\title{
Synthesis of Molecular 2D Materials via Low-energy Electron Induced Chemical Reactions
}

\author{
Andrey Turchanin ${ }^{\star a b c}$
}

\begin{abstract}
After the demonstration of a variety of inorganic two-dimensional (2D) materials (graphene, hBN, MoS etc.), molecular 2D materials have attracted a significant research interest as well. However, the direct synthesis of these materials is an exceptionally challenging task for chemists. In this review article, a simple and robust physical method for the synthesis of molecular 2D materials is presented based on low-energy electron induced chemical reactions in aromatic molecular layers. In this way, ultrathin $(\sim 1 \mathrm{~nm})$ molecular nanosheets with adjustable chemical and physical properties called Carbon Nanomembranes (CNM) can be prepared. Moreover, the method enables the synthesis of various other 2D organic-inorganic hybrids (e.g. MoS $-\mathrm{CNM}$, graphene-CNM lateral heterostructures, etc.) or $\sim 20 \mathrm{~nm}$ thick nanosheets of organic semiconductors. Mechanisms of the reaction and functional properties of these molecular 2D materials including their chemical functionalization and engineering of hybrid hierarchical structures for application in nanoscience and nanotechnology are discussed in this article.
\end{abstract}

Keywords: 2D materials · Carbon nanomembrane · Electron irradiation induced chemistry · Molecular nanosheet $\cdot$ Organic semiconductor

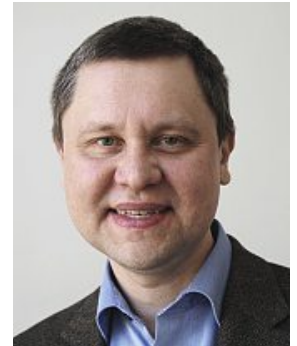

Andrey Turchanin studied physics and materials science at the National University of Science and Technology (MISIS), Moscow (PhD 1999). In 2000 he moved to the University of Karlsruhe with an Alexander von Humboldt Fellowship. 2004-2014 he joined the Faculty of Physics at the University of Bielefeld where he completed his habilitation in Experimental Physics in 2010. In 2012 Turchanin was awarded a Heisenberg Fellowship of the German Research Foundation (DFG) and in 2013 the Bernhard-Heß-Prize of the University of Regensburg for his research in the field of emerging 2D materials. Since 2014 he is a professor of physical chemistry at the Friedrich Schiller University Jena, where he is leading the group of 'Applied Physical Chemistry \& Molecular Nanotechnology'.

\section{Introduction: Materials Synthesis with Electron Beams}

Radiation-induced chemistry deals with the material modification under irradiation with photons (X-rays, $\gamma$-rays, UV irradiation), subatomic particles ( $\alpha$-particles, $\mathrm{He}^{+}$-ions, $\beta$-particles, neutrons, etc.) or a mixture thereof. ${ }^{[1]}$ This area of research can be dated back at least to the discovery by Wilhelm Conrad Röntgen in 1895 of the irradiation that he named X-rays, and which, as he reported, ${ }^{[2]}$ could modify photographic plates even if packaged in a carton envelope. Already this example demonstrated that radiation chemistry may induce some processes, which are hardly possible, if at all, using the traditional chemical approaches. Ionizing radiation typically has an energy of $10 \mathrm{eV}$ or more, which is higher than the ionization potential of most organic and inorganic compounds. Therefore it is suitable for the activation of various chemical processes without applying any additional treatment, e.g., by temperature or by introduction of catalysts.

Among the different types of ionizing radiation, electron irradiation takes a special place. Electron beams can be generated with adjustable energies as well as be focused, defocused or deflected by relatively simple technical means. This combination of properties makes the electron beam a flexible tool for materials synthesis and nanofabrication. Thus, the focused electron beams used, for example in lithography, enable the fabrication of devices and components for nanoelectronics, nanophotonics and nanomechanics with an unprecedented high lateral resolution and complexity (see e.g. ref. [3]). On the other hand, large-area irradiation of the materials with defocused electron beams induces their functional modification including polymer curing, surface hardening or microbiological sterilization (see e.g. ref. [4]).

Application of the electron beams in materials processing relies on scission of existing chemical bonds and formation of new ones. The related mechanistic details depend very much on the energy of the employed electron beams. In case of the interaction of a primary high-energy electron (typically tens of $\mathrm{keV}$ and higher) with a molecule, the momentum is directly transferred to the atomic nuclei, which may result in rupture of the existing and formation of a new chemical bond. ${ }^{[5]}$ In this way, the synthesis of a variety of novel carbon-based nanomaterials including carbon onions, ${ }^{[6]}$ carbon nanotubes, ${ }^{[7]}$ graphene nanoribbons ${ }^{[8]}$ or functionalized single layer graphene sheets ${ }^{[9]}$ was demonstrated. On the other hand, a primary low-energy electron (typically significantly below $1 \mathrm{keV}$ ) interacts directly with the molecular orbital resulting in its electronic excitation. ${ }^{[10-12]}$ Then the electronic excitation can trigger a modification of the chemical bond, which leads to dissociation of the pristine and formation of new molecular compounds culminating even in extended molecular networks (see e.g. refs. [13-16]). 
In this article, a simple and robust method for the room temperature and solvent-free synthesis of two-dimensional (2D) carbon-based materials via low-energy electron beam induced chemical reactions in organic monolayers and thin films will be reviewed. Fig. 1 shows a photograph of the high vacuum chamber (base pressure $2 \times 10^{-8}$ mbar) of a low-energy electron beam reactor (LEEBR), which can be employed to this end. Two main components of the LEEBR are recognized through the window. These are an electron flood gun with an adjustable energy of $10-1000 \mathrm{eV}$ and a sample table with integrated Faraday cups enabling a measurement of the electron dose during irradiation. In the following sections it will be demonstrated that such a simple experimental set-up enables the synthesis of a manifold of functional molecular 2D materials including multifunctional molecular nanosheets with $\sim 1 \mathrm{~nm}$ thickness ${ }^{[17,18]}$ (section 2), nanosheets of organic semiconductors ${ }^{[19]}$ (section 3 ) as well as atomically thin free-standing lateral organic-inorganic heterostructures (section 4). ${ }^{[20]}$ In section 5 these results will be summarized and an outlook will be presented for implementation of the developed synthetic methods in nanotechnology.

\section{Carbon Nanomembranes (CNMs)}

In this part, the synthesis of molecular nanosheets - carbon nanomembranes (CNM) - will be introduced,[17,18] (Fig. 2). The process is based on the electron irradiation-induced crosslinking of aromatic self-assembled monolayers, which was discovered by Gölzhäuser et al.[14] for 1,1'-biphenyl-4-thiol (BPT) self-assembled monolayers (SAMs) on gold. Low-energy electron irradiation of the BPT SAMs in high vacuum, with typical electron energies of $50-100 \mathrm{eV}$, results in rupture of the $\mathrm{C}-\mathrm{H}$ bonds in biphenyl molecules leading to a crosslinking between their adjacent aromatic rings. As a dominating mechanism for this chemical transformation, dissociative electron attachment (DEA) was suggested. ${ }^{[16]}$ In DEA, ${ }^{[10-12]}$ an impinging electron is captured by a molecular orbital forming a transition negative ion, the time evolution of which causes a bond rupture. As demonstrated by

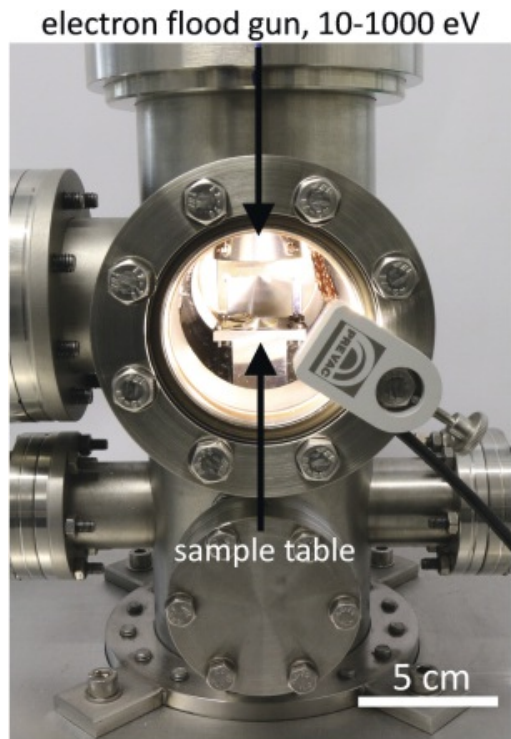

Fig. 1. Photograph of the vacuum chamber of a homemade low-energy electron beam reactor (LEEBR-3) at the Friedrich Schiller University Jena. An electron flood gun and a sample table can be recognized through the window; black arrows are guiding your eye. The LEEBR-3 enables a homogenous irradiation of the samples with dimension up to $30 \mathrm{~mm} \times 30 \mathrm{~mm}$ using electron energies of 10-1000 eV. The electron dose is measured during the irradiation by Faraday cups, which are located on the sample table. The residual pressure in the chamber during the irradiation is $\sim 2 \times 10^{-8}$ mbar. A typical time for the irradiation of a sample with $50 \mathrm{eV}$ electrons and a dose of $50 \mathrm{mC} / \mathrm{cm}^{2}$ is $\sim 10 \mathrm{~min}$. The photograph is by Christof Neumann, Friedrich Schiller University Jena.
UV photoelectron spectroscopy, quantum chemical and molecular dynamics calculations of BPT SAMs on gold, the formation of single- and double-links (carbon-carbon bonds) between phenyl rings of the molecules is expected during the crosslinking. ${ }^{[16]}$ This picture is supported also by a UV-Vis spectroscopic study of the refractive indexes of the monolayers. ${ }^{[21]}$ Moreover, recent molecular dynamic calculations suggest[22] that a partial dissociation of the aromatic rings can also take place in the BPT SAM upon this process, which is in agreement with some desorption of carbon observed by X-ray photoelectron spectroscopy (XPS). ${ }^{[23]}$ The XPS data also show that the electron irradiation-induced crosslinking results in the formation of new sulphur species and reconstruction of the sulphur/gold interface. ${ }^{[16]}$ These structural changes of the interface are in agreement with the low-electron energy diffraction (LEED) and scanning tunneling microscopy (STM) results that demonstrate a loss of the long-range order in the SAMs upon crosslinking. ${ }^{[24,25]}$ Note that not only primary electron irradiation but also secondary electrons (SE) produced in the substrate contribute to the crosslinking process. ${ }^{[26]}$ For BPT SAMs, the total electron dose required to induce a complete crosslinking is $\sim 50$ $\mathrm{mC} / \mathrm{cm}^{2}{ }^{[16]}$ Taking into account the surface coverage of BPT SAMs on gold and the respective SE yield, the cross-section of this process was estimated as $\sim 10^{-18} \mathrm{~cm}^{2}$. ${ }^{[16]}$ In summary, the lowenergy electron irradiation of a BPT SAM on gold results in the crosslinking of the monolayer. This process converts a BPT SAM into a covalently bonded, short-range ordered molecular network, i.e. into a molecular nanosheet, which has been introduced above as a carbon nanomembrane (CNM).

The formed BPT CNM possesses an extraordinary mechanical ${ }^{[27]}$ and thermal stability. ${ }^{[28]}$ Already in 2005 it has been shown ${ }^{[27]}$ that BPT CNMs with only $\sim 0.9 \mathrm{~nm}$ thickness can easily be prepared as free-standing sheets spanning openings with lateral dimensions over $30 \mu \mathrm{m}$. Furthermore BPT CNMs with extremely large free-standing lateral dimensions over $\sim 0.5 \mathrm{~mm}$ were demonstrated recently. ${ }^{[29]}$ A typical helium ion microscope (HIM) image of a BPT CNM lying on a hexagonal metal grid is presented in Fig. 3a. To enable the fabrication of such free-standing CNM structures as well as their van der Waals heterostructures, Nottbohm, Beyer, Gölzhäuser and co-workers have developed a flexible transfer method. ${ }^{[23,30-33]}$ This method employs a supportive poly(methyl methacrylate) layer to transfer the CNMs from their growth substrates onto new solid or holey target substrates. This method, known in the literature as PMMA-assisted transfer, is now applied widely in the 2D materials community to transfer any kind of 2D material.[34]

In 2007 it was found that BPT CNMs sustain also extremely high annealing temperatures in vacuum, ${ }^{[28]}$ which enabled Turchanin et al. to develop a new method for scalable synthesis of graphene monolayers with tunable crystallinity and electronic properties. ${ }^{[24,32,35-37]}$ Using this method and employing area selective electron irradiation of BPT SAMs, graphene monolayers with predefined shapes can be grown. ${ }^{[38,39]}$ These sculptured graphene structures can be transferred onto various target substrates and prepared as free-standing sheets preserving their integrity. ${ }^{[39]}$

A systematic study of the response of various aromatic SAMs (oligophenyls, hexaphenylbenzene, polycyclic aromatic hydrocarbons, etc.) towards low-energy electron irradiation has revealed that CNMs can be synthesized from a variety of molecular compounds. ${ }^{[23]}$ In this way, the chemical and physical properties of CNMs can be tuned. Thus, the thickness of individual CNMs can be adjusted from $\sim 0.5$ to $3 \mathrm{~nm}$. The surface structure of the formed SAMs and molecular shape of the SAM building compounds predefines the presence or absence of nanopores in the $\mathrm{CNMs}^{[23]}$ enabling the synthesis of nanomembranes for ultrafiltration applications. ${ }^{[25,40]} \mathrm{By}$ an appropriate choice of the molecular precursors, functional groups can be introduced allowing their flexible chemical or biochemical functionalization for 


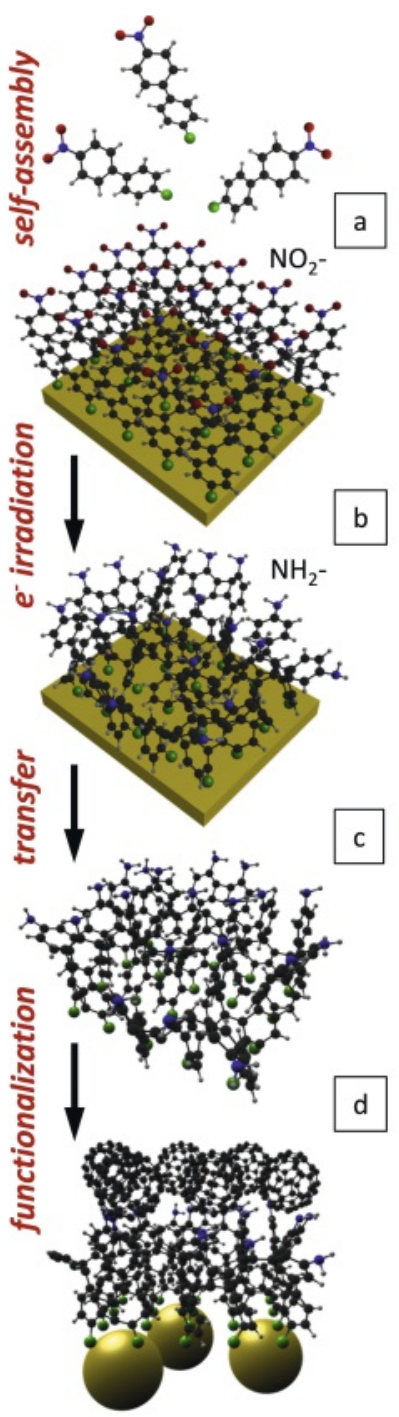

Fig. 2. Schematic representation of the carbon nanomembrane (CNM) synthesis by low-energy electron irradiation of aromatic self-assembled monolayers. a) A SAM of aromatic molecular compounds formed on a substrate; here, a 4'-nitro-1,1'-biphenyl-4-thiol (NBPT) SAM on gold is depicted. b) Low-energy electron irradiation induces the lateral crosslinking and converts the NBPT SAM into a molecular nanosheet - CNM. In case of the NBPT SAMs, also the terminal nitro groups are converted into amino groups. c) The CNM possesses a high mechanic stability and can be prepared as a free-standing nanosheet. In the presented example, the free-standing nanosheet forms a Janus CNM with one face terminated with amino groups ( $\mathrm{N}$-face) and another face terminated with sulphur containing groups (S-face). d) The $\mathrm{N}$-face and S-face of the Janus CNMs can be independently and specifically functionalized, for example, as schematically shown here, by $\mathrm{C}_{60}$ on the $\mathrm{N}$-face and gold nanoparticles on the S-face. applications in energy conversion and nanobiotechnology.[41-43] The mechanical or dielectric properties of CNMs can be tuned in this way as well, ${ }^{[44,45]}$ enabling their integration into various electronic devices as complementary 2D dielectric material (e.g. nanosensors, ${ }^{[43]}$ nanocapacitors ${ }^{[46]}$, protective (anticorrosive) coatings ${ }^{[39,47]}$ or as an active material in a nanomechanical system. ${ }^{[32,48]}$

A comprehensive review on the CNM properties and their prospects for applications in nanoscience and nanotechnology has been recently presented by Turchanin and Gölzhäuser. ${ }^{[18]}$ Therefore, in this section, only two more recent examples will be presented, which relate to the unique possibility for chemical functionalization of $\mathrm{CNMs}^{[49]}$ and their microfabrication into free-standing 2D nanostructures. ${ }^{[50]}$

\subsection{Chemical Functionalization of CNMs}

The possibility to introduce chemically active groups on both surfaces of the otherwise chemically inert CNMs opens up a variety of possibilities for their post-functionalization and creation of functional hybrid 2D nanosheets. ${ }^{[41,42,49,51]}$ As schematically shown in Fig. 2 for an example of the 4'-nitro-1,1'-biphenyl-4-thiol (NBPT) SAM on gold, the electron irradiation induces not only the SAMs crosslinking into a CNM with $\sim 1 \mathrm{~nm}$ thickness, but it also converts the terminal nitro groups into amino groups ${ }^{[15]}$ (Fig. 2a,b). Transferred from the substrate and suspended freely on a holey structure, both faces with different chemically active groups are available for the specific functionalization (Fig. 2c). In the resulting molecular nanosheet one face (N-face) is terminated with amino groups and the opposite face (S-face) with the sulphur-containing groups (thiols and sulphides). ${ }^{[42,49]}$ Such a bifacial CNM can be referred as a Janus CNM.

Zheng et al. used Janus CNMs for the engineering of molecular nanosheets with efficient energy-funneling properties. ${ }^{[42]}$ To this end, two different fluorescent dyes - tetramethylrhodamine isothiocyanate (TMR) and ATTO647N-maleimide - were grafted on the $\mathrm{N}$-face and the $\mathrm{S}$-face of a Janus CNM, respectively. Due to the distance of only $\sim 1 \mathrm{~nm}$ between the donor TMR and the acceptor ATTO647N, which is defined by the CNM thickness, an effective fluorescence resonance energy transfer (FRET) was demonstrated, showing the nanomembranes functionality. ${ }^{[42]}$

In general, Janus CNMs provide a universal platform for chemistry in two dimensions and in particular for the generation of complex hierarchical systems consisting not only of organic but also of organic/inorganic nanomaterials. Fig. $2 d$ schematically shows a low-dimensional hybrid assembled on a Janus CNM functionalized on its $\mathrm{S}$-face with gold nanoparticles and the $\mathrm{N}$-face with $\mathrm{C}_{60}$ fullerenes. These hybrids were engineered and studied with respect to their mechanical properties and potential for assembling van der Waals (vdW) heterostructures (see e.g. ref. [52]), which consist of $0 \mathrm{D}$ and $2 \mathrm{D}$ materials. ${ }^{[49]}$ Fig. $3 \mathrm{~b}$ shows a HIM image of Janus CNMs with immobilized $\sim 16 \mathrm{~nm}$
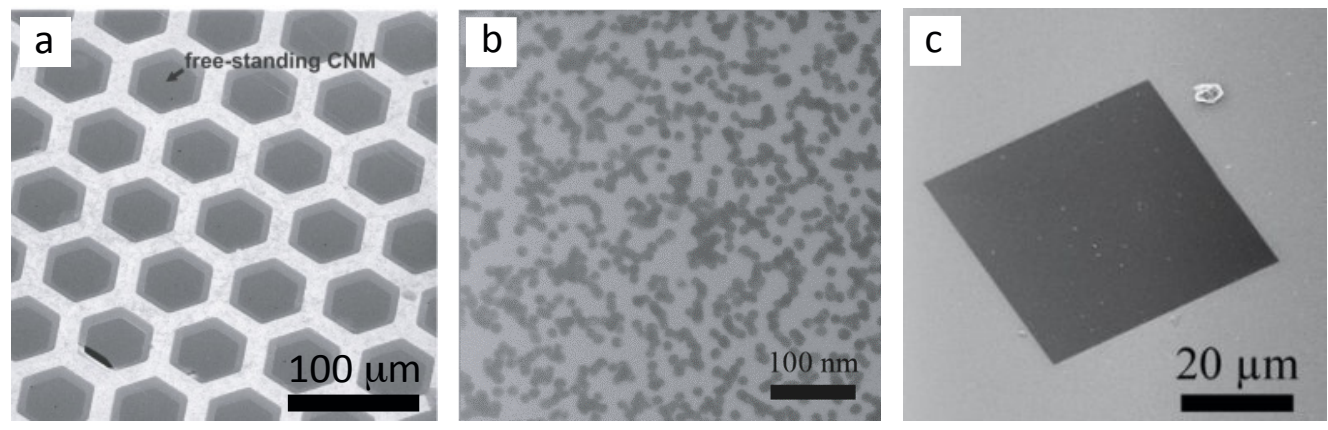

Fig. 3. Helium ion microscope (HIM) images of free-standing CNMs. a) Large-area CNM with $\sim 1 \mathrm{~nm}$ thickness spanning a hexagonal metal grid. ${ }^{[29]}$ b) Janus CNM on a metal grid with $16 \mathrm{~nm}$ gold nanoparticles immobilized on its S-face. $\left.{ }^{[49]} \mathrm{C}\right) \mathrm{A}$ van der Waals heterostructure consisting of four layers: (Janus CNM)/(C60-Janus $\mathrm{CNM})_{3}$ - spanning an orifice in a SiN membrane with dimensions of $40 \times 44 \mu \mathrm{m}^{2}$. 
gold nanoparticles. These nanoparticles cover $\sim 50 \%$ of the nanomembrane surface demonstrating their homogenous and effective integration into the hybrid. Fig. $3 \mathrm{c}$ presents an image of a vdW heterostructure consisting of four 2D layers stacked on each other - $($ Janus CNM $) /\left(\mathrm{C}_{60} \text {-Janus } \mathrm{CNM}\right)_{3}$ - over an orifice with dimensions of $40 \times 44 \mu \mathrm{m}^{2}$. Despite their multiple nanosheet structure and the functionalization with $\mathrm{C}_{60}$, the resulting nanomembrane is homogeneous. Additionally, it does not show a degradation of the mechanical properties in the bulge test ${ }^{[49]}$ with respect to the individual nanosheets, revealing a Young's modulus of $8.7 \pm 1.5 \mathrm{GPa}$.

The presented examples demonstrate that the flexible chemical functionalization of one or both faces of a CNM provides a universal and powerful platform towards the generation of novel functional hybrid 2D materials and their vdW heterostructures. This flexibility distinguishes CNMs from many other 2D materials, in particular from the inorganic ones. It paves the way for their implementation in nanosystems for artificial photosynthesis, biofunctional membranes for tissue engineering and molecular recognition, nanomembranes for osmosis and water desalination applications, or as active materials in nanosensors and photoelectrical devices, etc.

\subsection{Free-standing Nanopatterned CNMs}

Due to the very favorable mechanical properties including the Young's modulus of $\sim 10 \mathrm{GPa}^{[32,44]}$ and the tensile strength of $\sim 500 \mathrm{MPa},{ }^{[53]}$ not only continuous free-standing CNMs, as shown in Fig. 3, but also nanostructured free-standing CNMs can be manufactured efficiently, ${ }^{[33,50]}$ (Fig. 4). The use of EUV interference lithography ${ }^{[54]}$ (EUV-IL) is particular promising for the nanopatterning of CNMs, as it allows both the formation of CNM nanostructures via the direct EUV-induced crosslinking ${ }^{[26,33]}$ or the implementation of standard photoresist-based microfabrication techniques. ${ }^{[50]}$ In both cases, nanostructures with lateral dimension down to $20 \mathrm{~nm}$ have been demonstrated in free-standing CNMs by Turchanin et al. ${ }^{[33,50]}$ Using a wavelength of $13.5 \mathrm{~nm}$, EUV-IL potentially enables a large area, highly parallel and highthroughput nanopatterning with a sub10 nm resolution. ${ }^{[54,55]}$

Fig. 4a shows free-standing $1 \mathrm{~nm}$-thick CNM nanoribbons of $150 \mathrm{~nm}$ width prepared by the EUV-IL induced pattern on a continuous CNM and afterwards transferred onto a TEM grid with a holey lacey carbon support film. ${ }^{[50]}$ The total size of this pattern is $\sim 1 \times 1 \mathrm{~mm}^{2}$. A parallel array of the nanoribbons on the flat support (bright) and the freestanding nanoribbons spanning the open areas (dark) can be clearly recognized in the HIM image; the inset shows a high magnification image of the free-standing nanoribbons. A $1 \mathrm{~nm}$-thick CNM nanomesh with $260 \pm 5 \mathrm{~nm}$ wide openings and $90 \pm 5 \mathrm{~nm}$ wide mesh lines is shown in Fig. 4b. It demonstrates the remarkable mechanical stability of an intact CNM free-standing area of $2500 \mu \mathrm{m}^{2}$ (with exception of a few point defects) supported by a $50 \mu \mathrm{m} \times 50 \mu \mathrm{m}$ metal grid. Note that in all HIM images, free-standing CNM areas appear darker in comparison to the supported areas, which can be especially well recognized in the inset to Fig. $4 \mathrm{a}$, showing an increase of contrast towards the center of the free-standing nanoribbons. These imaging effects are related to the dielectric properties of the CNMs resulting in their electrostatic charging upon imaging with ions and thereby with a lower yield of secondary electrons reaching the detector of the HIM.[29]

The presented examples demonstrate that the application of EUV-IL on the nanopatterning of CNMs results in perforated $\sim \mathrm{nm}$ thick molecular nanosheets and nanoribbons, which can be prepared as free-standing objects on holey supports. This methodology opens up manifold possibilities of implementing the supported or free-standing nanopatterned CNMs in devices such as nanomechanical, nanophotonic and nanoelectrical systems, as well as their integration into lateral heterostructures with other 2D materials, which will be presented in section 4 of this review.
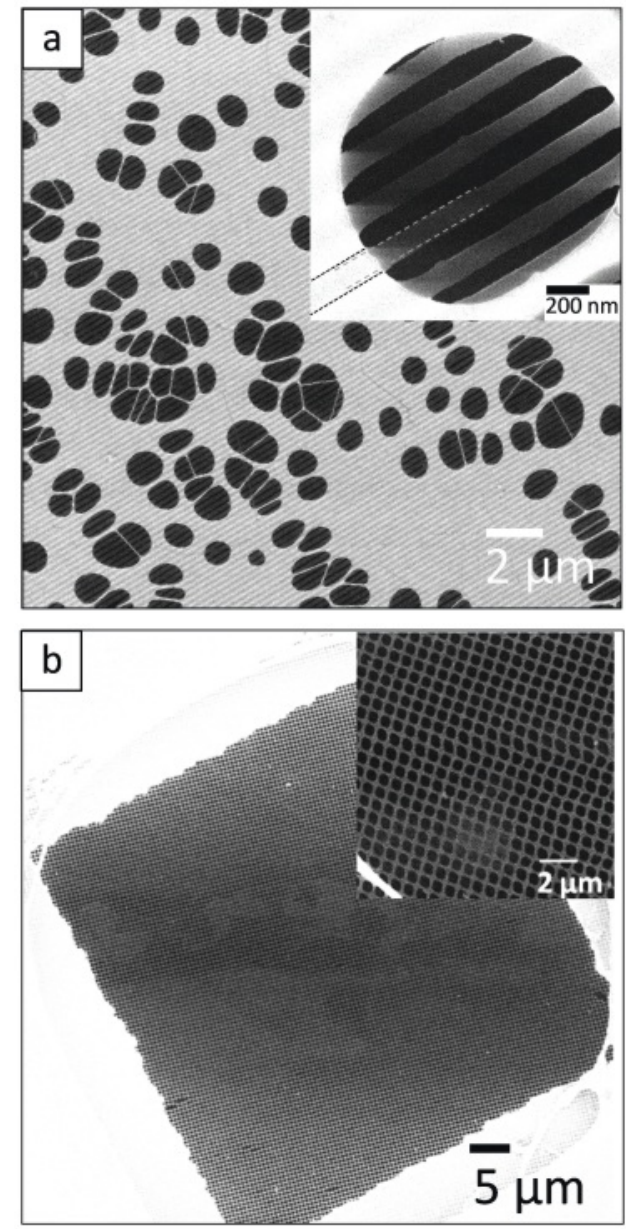

Fig. 4. Helium ion microscope (HIM) images of free-standing nanopatterned CNMs. ${ }^{[50]}$ a) $150 \pm 5 \mathrm{~nm}$ wide $\mathrm{CNM}$ nanoribbons transferred onto a TEM grid with a holey lacey carbon film. Free-standing areas appear darker in comparison to the lacey carbon substrate. Insert presents a magnified area with the free-standing nanoribbons. The free-standing nanoribbons appear narrower in comparison to the supported nanoribbons on the lacey carbon substrate. Dashed lines are included to guide the eye. b) A square shaped CNM nanomesh of $260 \pm 5 \mathrm{~nm}$ wide openings and $90 \pm 5 \mathrm{~nm}$ wide mesh lines on a $50 \times 50 \mu \mathrm{m}^{2}$ wide opening of a metal grid. The complete sample homogeneously covers about $1 \times 1 \mathrm{~mm}^{2}$. In the insert the mesh is magnified.

\section{Nanosheets of Organic Semiconductors}

In 2017 Nickel and Turchanin presented a method for the synthesis of free-standing nanosheets of organic semiconductors (OSC) via low-energy electron irradiation-induced crosslinking of the surface of OSC films, ${ }^{[19,56]}$ (Fig. 5a). This approach relies on a very basic property of electrons to penetrate into matter up to a certain depth, which depends on their kinetic energy (see e.g. ref. [57]). In this way, the crosslinking depth in OSC films can be adjusted with a precision of a few nanometers. The formed surface crosslinked layer, schematically shown in Fig. 5a as a black color gradient, stabilizes mechanically the whole OSC film and enables its transfer as a nanosheet onto new solid or holey substrates without disintegration as well as in the absence of any additional transfer medium. ${ }^{[19,56]}$ Fig. 5b shows a HIM image of such a $20 \mathrm{~nm}$ thick pentacene nanosheet transferred on an oxidized silicon wafer. This nanosheet was produced by irradiation with $500 \mathrm{eV}$ electrons and a dose of $3 \mathrm{mC} / \mathrm{cm}^{2}$ on the respective $20 \mathrm{~nm}$ thick pentacene film prepared by vacuum deposition. It is noticeable that some wrinkles are present in the topography indicating the sheet character of the transferred pentacene. The characteristic terrace-like topography of pentacene is conserved after irradiation and transfer. In Fig. 5c, an optical microscopy image of a $50 \mathrm{~nm}$ thick free-standing pentacene nanosheet spanning a hole with a 

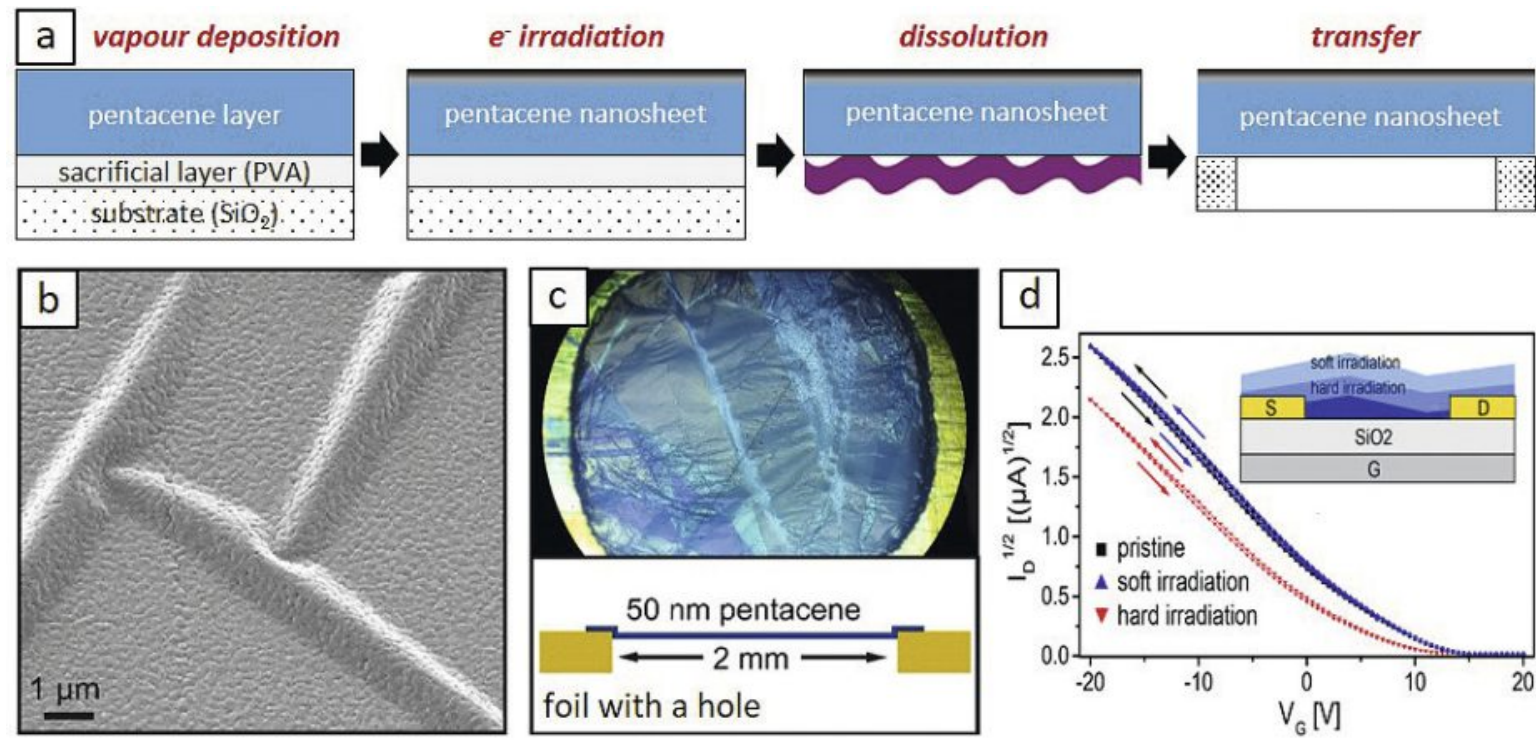

Fig. 5. Free-standing nanosheets of organic semiconductors (OSC). a) Schematic representation of the synthesis of pentacene nanosheets. First, an OSC film is prepared on a substrate, e.g. by vapor deposition, preferentially on a sacrificial layer such as poly(vinyl alcohol) (PVA). Then, the surface of the OSC layer is crosslinked by low-energy electron irradiation. Next, the sacrificial layer is dissolved and the OSC nanosheets, stabilized by the crosslinked surface, can be transferred onto a target substrate. b) $\mathrm{HIM}$ image of a crosslinked $20 \mathrm{~nm}$ pentacene nanosheet transferred onto a SiO${ }_{2}$ substrate. $\left.{ }^{[19]} \mathrm{C}\right)$ Optical microscope image of a $50 \mathrm{~nm}$ thin pentacene nanosheet spanned over a $2 \mathrm{~mm}$ hole in a $0.5 \mathrm{~mm}$ brass sheet. $\left.{ }^{[19]} \mathrm{d}\right)$ Electric transfer curves of a FET with pristine, partially crosslinked and strongly crosslinked surface layer. The arrows indicate the sweep direction. Inset: Schematic of the transistor. When irradiated softly (low-energy electrons), only the top layers of the pentacene film are crosslinked, i.e. the conduction channel at the dielectric interface is not affected. After hard irradiation (high-energy electrons), the crosslinked region penetrates the conduction channel and the device performance is reduced. ${ }^{[19]}$

diameter of $2 \mathrm{~mm}$ is shown. A detailed structural analysis of these nanosheets by X-ray diffraction demonstrated[19] that this remarkable mechanical stability results from lateral crosslinking of only the topmost 3-4 pentacene monolayers, which corresponds to a depth of $\sim 5 \mathrm{~nm}$. The remaining pentacene layers in the nanosheet preserve their original crystalline structure and therefore can be used for functional photonic or electronic applications. ${ }^{[19]}$

In Fig. 5d the transfer characteristics of a microfabricated field-effect transistor (FET) device from a pentacene nanosheet prepared by electron irradiation with $350 \mathrm{eV}$ is shown (blue triangles). The pentacene nanosheet does not show any degradation of the electronic properties in comparison to the non-irradiated pentacene film (black squares). Only a pentacene nanosheet prepared by irradiation with a higher energy of $700 \mathrm{eV}$ (red triangles) and therefore with a higher electron penetration depth, shows some reduction of the electronic properties. Moreover, it has been found that bottom contact pentacene FETs made from the transferable nanosheets are superior to the analogous devices prepared by direct vapor deposition of the pentacene films. Their contact resistance is about two orders of magnitude smaller due to a smoother interface with the gold contacts. ${ }^{[19]}$

This developed methodology opens up new possibilities towards the fabrication of organic semiconductor devices with transferable OSC nanosheets from a variety of aromatic molecules. ${ }^{[56]}$ It paves the way towards free-standing organic fieldeffect devices, an area which was reserved for single crystals so far, as well as to their integration with other 2D materials like graphene or transition metal dichalcogenides in heterostructure devices.

\section{2D Lateral Organic-Inorganic Heterostructures}

Low-energy electron irradiation allows not only to induce crosslinking between organic aromatic compounds, but also, as demonstrated by Winter et al., ${ }^{[20]}$ to stitch 2D organic and inorganic materials into their lateral heterostructures. This unique material composite is enabled by a combination of inorganic $2 \mathrm{D}$ sheets grown by chemical vapor deposition (CVD) with standard microfabrication techniques, molecular self-assembly and low-energy electron-induced chemical reactions. Fig. 6 shows two examples of graphene-CNM $(\mathrm{a}, \mathrm{b})$ and $\mathrm{MoS}_{2}-\mathrm{CNM}(\mathrm{c}, \mathrm{d})$ lateral heterostructures synthesized in this way. Initially both graphene and $\mathrm{MoS}_{2}$ monolayers were grown by CVD on copper ${ }^{[43]}$ or $\mathrm{SiO}_{2}^{[58]}$ substrates, respectively. Next, the formed inorganic atomically thin sheets were transferred from their growth substrates onto $\mathrm{Au} / \mathrm{mica}$ substrates. In case of graphene sheets, a photolithographic pattering was applied, whereas no lithographic treatment was used for $\mathrm{MoS}_{2}$ samples, as they grow by CVD as arrays of micrometersized triangular single crystals. To synthesize lateral heterostructures with CNMs, the monolayers of aromatic biphenyl-thiols (BPT SAM or NBPT SAM, see section 2) were self-assembled on the gold areas free of graphene or $\mathrm{MoS}_{2}$, and the samples were irradiated with $100 \mathrm{eV}$ electrons at a dose of $50 \mathrm{mC} / \mathrm{cm}^{2}$. This treatment results not only in the formation of CNMs from the respective SAM areas (see section 2), but also in their stitching with the edges of the graphene or $\mathrm{MoS}_{2}$ sheets and ultimately in the formation of continuous 2D lateral heterostructures.

Fig. $6 \mathrm{~b}$ shows an optical microscope image of a grapheneCNM heterostructure transferred onto a $300 \mathrm{~nm} \mathrm{SiO} / \mathrm{Si}$ wafer. This heterostructure consists of an array of square $\left(2.5 \times 2.5 \mu \mathrm{m}^{2}\right)$ graphene dots embedded in a CNM matrix. The graphene areas appear darker, as they have a higher optical contrast in comparison to the surrounding CNM. ${ }^{[21]}$ The graphene-CNM sheet is continuous over a whole macrofabricated area, which corresponds to $10 \times 10 \mathrm{~mm}^{2}$ in total.

A rupture defect is visible in the right top corner enabling to differentiate clearly between the bare $\mathrm{SiO}_{2}$ surface and the heterostructure area. It has been demonstrated ${ }^{[20]}$ that these heterostructures possess a sufficient mechanical stability to be also prepared as free-standing nanomembranes. Such a free-standing $\mathrm{MoS}_{2}-\mathrm{CNM}$ heterostructure was imaged with high-resolution transmission electron microscopy (HRTEM) by Mohn and Kaiser, ${ }^{20]}$ (Fig. 6d). The stitching region between these two 2D materials - CNM and $\mathrm{MoS}_{2}$ - can be clearly recognized and appears to be atomically sharp, which demonstrates that the lateral heterostructures can be synthesized with a precision close to the atomic scale with a width of the boundary between the constituting 2D materials below 

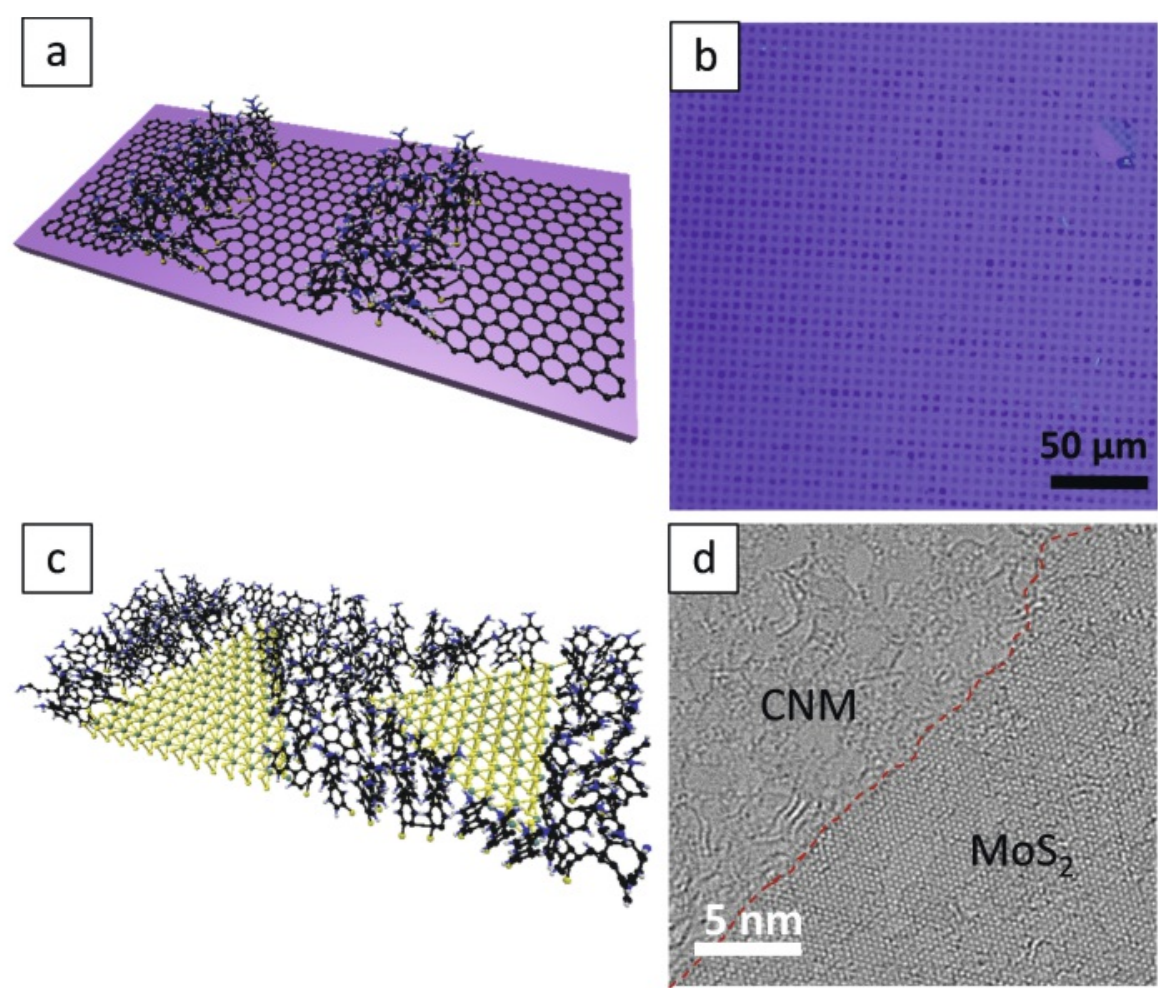

Fig. 6. 2D lateral organic-inorganic heterostructures synthesized by low-energy electron irradiationinduced stitching. ${ }^{[20]}$ a) Schematic of graphene-CNM heterostructure. b) Optical microscopy image of the heterostructure on a $300 \mathrm{~nm}$ $\mathrm{SiO}_{2} / \mathrm{Si}$ substrate. Graphene areas appears darker than CNM areas. The graphene square dots in (b) have a width of $2.5 \mu \mathrm{m}$. The whole sample has a size of $10 \times 10 \mathrm{~mm}^{2}$. c) Schematic of $\mathrm{MoS}_{2}-\mathrm{CNM}$ heterostructure. d) HRTEM image of the boundary between CNM (top left) and $\mathrm{MoS}_{2}$ (bottom right) in the free-standing heterostructure.
$2 \mathrm{~nm}$. In this process, low-energy electron irradiation activates the terminal atoms and chemical groups at the edges of graphene or $\mathrm{MoS}_{2}$ sheets resulting in the formation of new chemical bonds with the edges of the formed CNM in both heterostructure types.

The presented examples of hybrid organic-inorganic materials would be very difficult to realize by conventional chemical synthesis, which demonstrates the uniqueness of the applied synthesis by low-energy electron irradiation. This technique can induce chemical reactions in a variety of materials (see e.g. ref. [1]), and therefore can be applied potentially to a broad variety of organic and inorganic 2D materials. This physical approach enables the flexible synthesis of their lateral heterostructures with complex architectures incorporating some distinct electronic, optical and biochemical functions (due to the chemical functionalization of CNMs) into the atomically thin sheets. Therefore a broad panel of materials for nanoelectronic, nanophotonics and nanobiotechnology can be specifically engineered.

\section{Summary}

The synthesis of 2D materials beyond graphene has attracted significant research interest in the chemistry and materials science communities during the last years. ${ }^{[34,59-61]}$ Besides the activities on synthesis of different inorganic materials and their heterostructures, ${ }^{[52,62,63]}$ also organic 2D materials are coming more and more in focus. ${ }^{[17,18,64-66]} 2 \mathrm{D}$ materials consisting of individual organic molecules as their building blocks, provide an unprecedented flexibility for their tailored synthesis. The intrinsic molecular structure of the constituents, their organization in 2D assemblies and chemical functionalization paves a way towards the engineering of 2D materials with tunable electronic (dielectric, semiconductor, conductor), mechanical (hard or soft) and functional (catalytic, photoactive, biofunctional, etc.) properties. The applications of these synthetic materials in nanotechnology are manifold and include their implementation in molecular- and bio-recognition, energy storage, catalysis, photonics, electronics or ultrafiltration, just to name a few. However, direct preparation of molecular 2D materials by traditional chemical synthesis faces significant challenges. For instance, (i) the mechanism of many chemical reactions in the $2 \mathrm{D}$ constraint may differ significantly from the 3D analogues; (ii) the thermodynamically favorable 3D growth has to be avoided; (iii) implementation of the lateral organic and inorganic heterostructures materials can be very much restricted to a few material combinations.

As demonstrated in this review article, the low-energy electron beam induced synthesis of $2 D$ materials provides a simple and robust physical approach to address largely these challenges. It relates to the conversion of well-defined monolayers or ultrathin 2D films, prefabricated by physical or chemical deposition on solid substrates, into their nanosheets by irradiation induced crosslinking. As the impinging electrons possess sufficient energy to activate nearly any chemical bond, the crosslinking can be initiated within a variety of chemically dissimilar materials. Moreover, the activation on only specific chemical bonds may be tuned by an appropriate choice of the electron energy. ${ }^{[11]}$ The synthesis takes place at room temperature and does not require any solvents making it both energetically efficient and environmentally friendly. Moreover, all synthetic steps are compatible with clean room techniques, which enables their integration into the standard microfabrication technologies and processes as well as their scalability. As specific functional groups can be incorporated into the nanosheets, they can be modified further by conventional chemical functionalization. In this way, complex hierarchical 2D materials can be synthesized. Such a favorable combination of different options in the developed methodology opens broad avenues for its implementation in nanoscience and nanotechnology.

\section{Acknowledgements}

The author acknowledges all partners and collaborators, with whom he was lucky to work on the described topics since 2005, as well as the financial support of this research through the DFG, BMBF and EU funding agencies. Maria Küllmer is gratefully acknowledged for the proofreading.

Received: May 14, 2019

[1] A. Mozumder, Y. Hatano, 'Charged Particles and Photon Interaction with Matter: Chemical, Physicochemical, and Biological Consequences with Applications', Marcel Dekker: New York, 2004

[2] W. C. Röntgen, Ann. Phys. Chem. 1898, 65, 1.

[3] M. Feldman, 'Nanolithography: The Art of Fabricating Nanoelectronic and Nanophotonic Devices and Systems', Woodhead Publishing, 2014. 
[4] 'Industrial Accelerators and Their Applications', Eds R. W. Hamm, M. E. Hamm, World Scientific, 2012.

[5] A. V. Krasheninnikov, K. Nordlund, J. Appl. Phys. 2010, 107.

[6] D. Ugarte, Nature 1992, 359, 707.

[7] J. X. Li, F. Banhart, Nano Lett. 2004, 4, 1143.

[8] T. W. Chamberlain, J. Biskupek, S. T. Skowron, A. V. Markevich, S. Kurasch, O. Reimer, K. E. Walker, G. A. Rance, X. L. Feng, K. Müllen, A. Turchanin, M. A. Lebedeva, A. G. Majouga, V. G. Nenajdenko, U. Kaiser, E. Besley, A. N. Khlobystov, ACS Nano 2017, 11, 2509.

[9] A. Markevich, S. Kurasch, O. Lehtinen, O. Reimer, X. L. Feng, K. Müllen, A. Turchanin, A. N. Khlobystov, U. Kaiser, E. Besley, Nanoscale 2016, 8, 2711.

[10] E. Illenberger, Chem. Rev. 1992, 92, 1589.

[11] E. Illenberger, Surf. Sci. 2003, 528, 67.

[12] R. E. Palmer, P. J. Rous, Rev. Mod. Phys. 1992, 64, 383.

[13] J. Warneke, Z. Y. Wang, P. Swiderek, J. H. Bredehoft, Angew. Chem. Int. Ed. 2015, 54, 4397.

[14] W. Geyer, V. Stadler, W. Eck, M. Zharnikov, A. Gölzhäuser, M. Grunze, Appl. Phys. Lett. 1999, 75, 2401.

[15] W. Eck, V. Stadler, W. Geyer, M. Zharnikov, A. Gölzhäuser, M. Grunze, Adv. Mater. 2000, 12, 805.

[16] A. Turchanin, D. Käfer, M. El-Desawy, C. Wöll, G. Witte, A. Gölzhäuser, Langmuir 2009, 25, 7342

[17] A. Turchanin, A. Gölzhäuser, Prog. Surf. Sci. 2012, 87, 108.

[18] A. Turchanin, A. Gölzhäuser, Adv. Mater. 2016, $28,6075$.

[19] S. J. Noever, M. Eder, F. del Giudice, J. Martin, F. X. Werkmeister, S. Hallwig, S. Fischer, O. Seeck, N. E. Weber, C. Liewald, F. Keilmann, A Turchanin, B. Nickel, Adv. Mater. 2017, 29, 6.

[20] A. Winter, A. George, C. Neumann, Z. Tang, M. J. Mohn, J. Biskupek, N. Masurkar, A. L. M. Reddy, T. Weimann, U. Hübner, U. Kaiser, A. Turchanin, Carbon 2018, 128, 106.

[21] C. T. Nottbohm, A. Turchanin, A. Beyer, R. Stosch, A. Gölzhäuser, Small 2011, 7, 874 .

[22] A. Mrugalla, J. Schnack, Beilstein J. Nanotechnol. 2014, 5, 865.

[23] P. Angelova, H. Vieker, N.-E. Weber, D. Matei, O. Reimer, I. Meier, S. Kurasch, J. Biskupek, D. Lorbach, K. Wunderlich, L. Chen, A. Terfort, M. Klapper, K. Müllen, U. Kaiser, A. Golzhäuser, A. Turchanin, ACS Nano 2013, 7, 6489 .

[24] D. G. Matei, N.-E. Weber, S. Kurasch, S. Wundrack, M. Woszczyna, M. Grothe, T. Weimann, F. Ahlers, R. Stosch, U. Kaiser, A. Turchanin, Adv. Mater. 2013, 25, 4146.

[25] M. Ai, S. Shishatskiy, J. Wind, X. Zhang, C. T. Nottbohm, N. Mellech, A Winter, H. Vieker, J. Qiu, K.-J. Dietz, A. Gölzhäuser, A. Beyer, Adv. Mater 2014, 26, 3421.

[26] A. Turchanin, M. Schnietz, M. El-Desawy, H. H. Solak, C. David, A Gölzhäuser, Small 2007, 3, 2114

[27] W. Eck, A. Küller, M. Grunze, B. Völkel, A. Gölzhäuser, Adv. Mater. 2005 , $17,2583$.

[28] A. Turchanin, M. El-Desawy, A. Gölzhäuser, Appl. Phys. Lett. 2007, 90, 053102 .

[29] A. Beyer, H. Vieker, R. Klett, H. M. Z. Theenhausen, P. Angelova, A Gölzhäuser, Beilstein J. Nanotechnol. 2015, 6, 1712.

[30] A. Gölzhäuser, C. T. Nottbohm, A. Beyer, 'Method for transferring a monolayer', European Patent 2144711, US Patent 8377243 B2, 112007.

[31] C. T. Nottbohm, A. Beyer, A. S. Sologubenko, I. Ennen, A. Hütten, H. Rösner, W. Eck, J. Mayer, A. Gölzhäuser, Ultramicroscopy 2008, 108, 885.

[32] A. Turchanin, A. Beyer, C. T. Nottbohm, X. Zhang, R. Stosch, A. S. Sologubenko, J. Mayer, P. Hinze, T. Weimann, A. Gölzhäuser, Adv. Mater. 2009, 21, 1233

[33] M. Schnietz, A. Turchanin, C. Nottbohm, A. Beyer, H. H. Solak, P. Hinze, T. Weimann, A. Gölzhäuser, Small 2009, 5, 2651.

[34] A. C. Ferrari, F. Bonaccorso, V. Fal'ko, K. S. Novoselov, S. Roche, P. Boggild, S. Borini, F. H. L. Koppens, V. Palermo, N. Pugno, J. A. Garrido, R. Soran, A. Bianco, L. Ballerini, M. Prato, E. Lidorikis, J. Kivioja, C. Marinelli, T. Ryhänen, A. Morgurgo, J. N. Coleman, V. Nicolosi, L. Colombo, A. Fert, M. Garcia-Hernandez, A. Bachtold, G. F. Schneider, F. Guinea, C. Dekker,
M. Barbone, Z. Sun, C. Galiotis, A. N. Grigorenko, G. Konstantatos, A. Kis, M. Katsnelson, L. Vandersypen, A. Loiseau, V. Morandi, D. Neumaier, E. Treossi, V. Pellegrini, M. Polini, A. Tredicucci, G. M. Williams, B. H. Hing, J. H. Ahn, J. M. Kim, H. Zirath, B. J. van Wees, H. van der Zant, L. Occhipinti, A. Di Matteo, I. A. Kiinloch, T. Seyller, E. Quesnel, X. Feng, K. Teo, N. Rupesinghe, P. Hakonen, S. R. Neil, Q. Tannock, T. Löfwander, J. Kinaret, Nanoscale 2015, 7, 4598.

[35] A. Turchanin, D. Weber, M. Büenfeld, C. Kisielowski, M. V. Fistul, K. B. Efetov, T. Weimann, R. Stosch, J. Mayer, A. Gölzhäuser, ACS Nano 2011, 5 , 3896.

[36] A. Turchanin, Ann. Phys. 2017, 529, 1700168.

[37] A. Gölzhäuser, A. Turchanin, DE 102007041820 A1, EP 2188212 B1, US 9458019 B2 2007.

[38] C. T. Nottbohm, A. Turchanin, A. Beyer, A. Gölzhäuser, J. Vac. Sci. Technol. B 2009, 27, 3059 .

[39] N.-E. Weber, S. Wundrack, R. Stosch, A. Turchanin, Small 2016, 12, 1440.

[40] Y. Yang, P. Dementyev, N. Biere, D. Emmrich, P. Stohmann, R. Korzetz, X. Zhang, A. Beyer, S. Koch, D. Anselmetti, A. Gölzhäuser, ACS Nano 2018 , 12,4695 .

[41] A. Turchanin, A. Tinazli, M. El-Desawy, H. Großmann, M. Schnietz, H. H. Solak, R. Tampé, A. Gölzhäuser, Adv. Mater. 2008, 20, 471.

[42] Z. Zheng, C. T. Nottbohm, A. Turchanin, H. Muzik, A. Beyer, M. Heilemann, M. Sauer, A. Gölzhäuser, Angew. Chem. Int. Ed. 2010, 49, 8493.

[43] M. Woszczyna, A. Winter, M. Grothe, A. Willunat, S. Wundrack, R. Stosch, T. Weimann, F. Ahlers, A. Turchanin, Adv. Mater. 2014, 26, 4831.

[44] X. Zhang, C. Neumann, P. Angelova, A. Beyer, A. Gölzhäuser, Langmuir 2014, 30, 8221

[45] X. H. Zhang, E. Marschewski, P. Penner, A. Beyer, A. Gölzhäuser, J. Appl. Phys. 2017, 122, 7 .

[46] X. Zhang, E. Marschewski, P. Penner, T. Weimann, P. Hinze, A. Beyer, A. Gölzhäuser, ACS Nano 2018, 12, 10301.

[47] C. Nottbohm, A. Turchanin, A. Gölzhäuser, Z. Phys. Chem. 2008, 222, 917.

[48] X. Zhang, R. Waitz, F. Yang, C. Lutz, P. Angelova, A. Gölzhäuser, E. Scheer, Appl. Phys. Lett. 2015, 106, 5.

[49] Z. Zheng, X. Zhang, C. Neumann, D. Emmrich, A. Winter, H. Vieker, W. Liu, M. Lensen, A. Gölzhäuser, A. Turchanin, Nanoscale 2015, 7, 13393.

[50] A. Winter, Y. Ekinci, A. Gölzhäuser, A. Turchanin, 2D Mater 2019, 6, 8.

[51] I. Amin, M. Steenackers, N. Zhang, A. Beyer, X. H. Zhang, T. Pirzer, T. Hugel, R. Jordan, A. Gölzhäuser, Small 2010, 6, 1623.

[52] A. K. Geim, I. V. Grigorieva, Nature 2013, 499, 419.

[53] X. Zhang, A. Beyer, A. Gölzhäuser, Beilstein J. Nanotechnol. 2011, 2, 826.

[54] V. Auzelyte, C. Dais, P. Farquet, D. Grützmacher, L. J. Heyderman, F. Luo, S. Olliges, C. Padeste, P. T. Sahoo, T. Thomson, A. Turchanin, C. David, H H. Solak, MEMS MOEMS 2009, 8, 021204.

[55] B. Päivänranta, A. Langner, E. Kirk, C. David, Y. Ekinci, Nanotechnology 2011, 22, 375302

[56] A. Turchanin, B. Nickel, WO 2017/153524 A1, 2017

[57] S. Tanuma, C. J. Powell, D. R. Penn, Surf. Interface Anal. 1994, 21, 165.

[58] A. George, C. Neumann, D. Kaiser, R. Mupparapu, T. Lehnert, U. Hübner, Z. Tang, A. Winter, U. Kaiser, I. Staude, A. Turchanin, J. Phys.: Mater. 2019, 2, 016001.

[59] D. Geng, H. Y. Yang, Adv. Mater. 2018, 30, 1800865.

[60] Z. Cai, B. Liu, X. Zou, H.-M. Cheng, Chem. Rev. 2018, 118, 6091.

[61] F. Xia, H. Wang, J. C. M. Hwang, A. H. C. Neto, L. Yang, Nat. Rev. Phys. 2019, $1,306$.

[62] W. Choi, N. Choudhary, G. H. Han, J. Park, D. Akinwande, Y. H. Lee, Mater Today 2017, 20, 116.

[63] S. Manzeli, D. Ovchinnikov, D. Pasquier, O. V. Yazyev, A. Kis, Nat. Rev. Mater. 2017, 2, 17033

[64] W. Wei, A. D. Schlüter, Macromol. Rapid Commun. 2018, 1800719.

[65] X. Feng, A. D. Schlüter, Angew. Chem. Int. Ed. 2018, 57, 13748.

[66] C. Neumann, D. Kaiser, M. J. Mohn, M. Füser, N.-E. Weber, O. Reimer, A. Gölzhäuser, T. Weimann, A. Terfort, U. Kaiser, A. Turchanin, ACS Nano 2019, DOI: $10.1021 /$ acsnano.9b03475. 